\title{
RETRIEVAL OF ARTICULATE OBJECTS FROM IMAGES AND VIDEO USING INVARIANT SIGNATURES
}

\author{
Ronald Alferez and Yuan-Fang Wang \\ \{ronald,yfwang\}@cs.ucsb.edu \\ Department of Computer Science \\ University of California at Santa Barbara
}

\begin{abstract}
In this paper, we propose a new method of retrieving multi-part, articulate objects from images and video. The scheme is particularly well suited for analyzing images and video for objects that can pose differently with possible shape deformation and articulated motion. The scheme involves computing an invariant signature for each segmented region in the image, in a manner that is insensitive to translation, rotation, scale, and shear. Using circular cross-correlation, these signatures can then be efficiently compared with that of user-defined regions of interest. Ambiguities between individual region matches are then resolved through relaxation labeling techniques. A final match is established when a collection of segmented regions conform to the query object, both in terms of local shape description and global structural relation. The scheme thus allows for articulated movement of object parts within the scene. The procedure is easy to implement, yet shows promising results in its ability to isolate interesting regions in images and video, to account for structural and relational constraints among regions, and to integrate both local shape and global structural information for a detailed examination of the scene in a way that is invariant to many visual variations.
\end{abstract}

\section{INTRODUCTION}

The enormous size of multimedia archives available has necessitated the employment of automated means of categorizing and retrieving images and video from digital libraries. Often, categorization schemes such as directory trees, or text annotations such as closed captioning are insufficient or may even be unavailable to assist in the query. It then becomes necessary to rely on methods that analyze the contents of the images and video to answer a query.

In this paper, we propose a novel method of retrieving images and video based on the content. In particular, the technique attempts to select relevant images or video by searching for multiple regions within the scene. The search is based both on the local shape and color similarity of image regions to components of the query objects, and on the structural relation among those image regions. For example, suppose we wanted to find all videos that featured the cartoon character Woody Woodpecker. To form the query, we could select the regions that comprise that character from a segmented sample image. The set of user-defined regions could then be collectively compared with the segmented regions of image frames from videos.

In many cases, for an image region to conform to a component of the query object, the shape (in addition to color) has to match. Such match requires detailed examination of shape traits of a region. Global methods, such as histograms, can be imprecise and can be easily influenced by changes in parts of the scene that are irrelevant (e.g., the background). This is especially true when the region of interest is merely a small component of the entire image. Moreover, relying solely on color information might produce too many false-positives, as the color composition of an image region might have some ambiguity with the rest of the scene. Furthermore, a single image region seldom describes complex objects, and hence, structural and relational information among multiple regions must be considered.

Our approach addresses the following issues: First, the images must be properly segmented to isolate objects or regions within the scene. We adopt a straightforward approach, by partitioning the image based on color gradients. Second, the silhouette of the object may be inadequate to describe the object and disambiguate it from the rest of the scene. Hence, the interior details of an object must also be analyzed. Third, the object may have articulated movement, such as moving of the arms and legs, or tilting of the head. The manner in which we circumvent this is by analyzing the object as a composition of multiple parts. This way, individual parts 
of the object can have localized articulated motion. Lastly, the object may pose differently in each scene, thus translation, rotation, scaling (zoom), and shear effects must be accounted for. In order to capture these visual distortions commonly associated with a changing set of camera parameters. The affine transform is used as an appropriate camera model, thus achieving viewpoint invariance.

The benefits of such a system can be significant. Children interested in some cartoon character can browse through thousands of videos that are of interest. Trademark or icon image databases can be searched in a detailed manner, by relying not only on color nor on shape boundary, but also on the shape of the parts that compose the entire object. In this fashion, images that contain only parts of the query can still be located. Videos can also be indexed by examining the composition of the characters in the scene, and not merely be based on the changing global parameters of entire frames.

\section{TECHNICAL DETAILS}

The retrieval problem is addressed by first segmenting the image into regions based on color gradients. The shape of each extracted region is then analyzed to produce an affine-invariant signature that is insensitive to translation, rotation, scale, and shear, caused by object motion and/or movement of the camera. Using circular cross-correlation, these signatures can then be efficiently compared with that of user-defined regions of interest. However, comparing regions individually can usually produce ambiguous matches. For example, a circular region can easily match to a wheel, a balloon, or even a face. Therefore, relaxation labeling that utilizes both the appearance of a region and its relations to its surrounding regions is employed to resolve ambiguities that can occur between individual matches. By matching the regions within the context of its surrounding, the search process then becomes more reliable.

\subsection{Image segmentation}

Segmentation is an extremely difficult problem and is currently the focus of much research. As such, the segmentation task here is dealt with only lightly; as the appropriate segmentation technique highly depends on the domain of the image or video library. For example, in the case of cartoon videos, it is usually possible to reliably segment the scene based solely on the different colors in the image. In more complicated scenarios, such as real videos, more sophisticated segmentation techniques, such as those based on texture analysis, may be needed.
The minimum requirement is that no segmented region can comprise more than one component of the scene (i.e., caused by under-segmentation). For example, a single region should not exclusively contain both the sun and the sky, if in fact one is interested in isolating the sun. In the ideal case, the segmentation should be performed consistently in both the query image and in the image/video library. This may be an unrealistic assumption. However, merging adjacent regions and computing new signatures for these composed regions can accommodate discrepancies caused by over-segmentation.

\subsection{Comparing individual image regions}

After the scene has been partitioned, an affine-invariant signature is computed for each region based on its boundary. This signature describes the region in a way that is insensitive to many types of visual distortions, such as translation, rotation, scale, and shear, due to both camera and object motion. The signature can then be used to efficiently compare it with regions of interest in the query through circular cross-correlation.

\section{Computing the Invariant signature}

Let $\boldsymbol{C}(t)=\left[\begin{array}{ll}\mathbf{x}(\mathrm{t}) & \mathbf{y}(\mathrm{t})\end{array}\right]^{\mathrm{T}}$ define the contour of a region. Tracing four points along the path of the curve enables the formation of an affine-invariant signature $f(t)$ :

$$
f(t)=\frac{\left|\begin{array}{ccc}
x_{t+i_{0}} & x_{t+i_{1}} & x_{t+i_{2}} \\
y_{t+i_{0}} & y_{t+i_{1}} & y_{t+i_{2}} \\
1 & 1 & 1
\end{array}\right|}{\left|\begin{array}{ccc}
x_{t+i_{1}} & x_{t+i_{2}} & x_{t+i_{3}} \\
y_{t+i_{1}} & y_{t+i_{2}} & y_{t+i_{3}} \\
1 & 1 & 1
\end{array}\right|}
$$

where $\mathrm{t}$ is some affine-invariant parameter along the curve, and $i_{k}, k=0,1,2,3$, are constants that represent index offsets from $t$. The behavior of the signature can be interpreted as its reaction to changes in the ratio of the signed area of the triangle formed by the first three points relative to that formed by the last three points (see Figure 1 ). The computed signature is invariant because of a wellknown geometric property on affine transform, which states that the ratio of the area of two triangles is constant under an affine transformation [5]. That is, given any two triangles $\Delta_{1}$ and $\Delta_{2}$,

$$
\frac{\alpha\left(\Delta_{1}\right)}{\alpha\left(\Delta_{2}\right)}=\frac{\alpha\left(\Delta_{1}^{\prime}\right)}{\alpha\left(\Delta_{2}^{\prime}\right)}
$$

where $\alpha$ denotes the area and $\Delta^{\prime}$ is some affine transformation of $\Delta$. In Eq.1, the formula uses the signed area of a triangle, which also takes into account the 
ordering of the three vertices. A derivation and analysis of a signature similar to Eq. 1 is offered in [1].

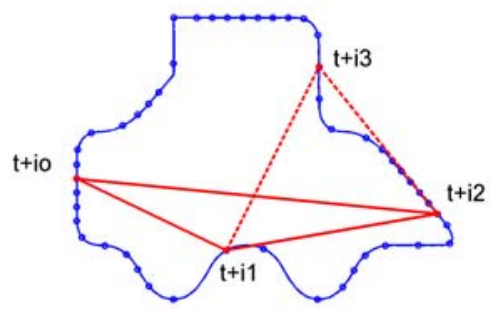

Figure 1. Computing the signature of a region using four points traveling along the boundary.

The two signatures $f_{1}$ and $f_{2}$ are then matched using circular cross-correlation:

$$
r_{x y}(l)=\frac{1}{N} \sum_{t=0}^{N-1} f_{1}(t) f_{2}(t+l)
$$

where $l=0,1,2, \ldots N$ - 1 , is a constant representing the phase shift (or lag) of one signature relative to the other. The equation $r_{x y}(l)$ is maximized to determine the correlation coefficient of the best alignment of the two signatures, thus accounting for the difference in the starting points between two contours.

\section{Affine-invariant parameterization.}

In general, the parameterization of curves such as the intrinsic arc length is not invariant to affine transformations. For instance, scaling the curve in one dimension changes the length of the curve along that axis. Thus, a consistent sampling along the curve must be established in an affine invariant manner. Two popular methods are described. In Eq. 4, the parameterization accounts for curvature to determine the sampling of the curve. In Eq. 5, the area of the enclosed contour is used to establish invariant parameterization. More details can be found in [2][3] describing the two methods:

$$
\begin{aligned}
& \tau=\int_{a}^{b} \sqrt[3]{\ddot{x} \ddot{y}-\ddot{x} \ddot{y}} d t \\
& \sigma=\frac{1}{2} \int_{a}^{b}|x \dot{y}-y \dot{x}| d t
\end{aligned}
$$

where $\dot{x}, \dot{y}$ are the first and $\ddot{x}, \ddot{y}$ are the second derivatives with respect to any parameter $t$ (possibly the arc length), and $(a, b)$ is the path along a segment of the curve.

\subsection{Incorporating relational constraints}

Matching individual regions can be and often is ambiguous, since the regions usually represent parts of a larger object. For example, a circle shaped region can be representative of many things, such as a wheel, the sun, or a head. Usually, the only way to disambiguate this is to also consider the adjacent regions as well.

Using probabilistic relaxation labeling techniques, ambiguities between individual matches can be resolved. All of the regions are identified simultaneously, not only by comparing individual regions, but also by matching it within the context of its neighbors. Examining how neighboring regions are matched can thus corroborate the correctness of a match for a region.

Let $\boldsymbol{R}$ be the set $\left\{r_{1}, r_{2}, \ldots r_{\mathrm{n}}\right\}$ of $n$ regions in the segmented image from a library. Let $\boldsymbol{L}$ be the set $\left\{l_{1}, l_{2}, \ldots\right.$ $l_{\mathrm{m}}$ \} of $m$ possible matching regions defined by the user in the query. Define $\boldsymbol{P}_{i}\left(\boldsymbol{l}_{k}\right)$ to be the probability that region $\boldsymbol{r}_{\mathrm{i}}$ is a correct match for query region $\boldsymbol{l}_{\boldsymbol{k}}$. Relaxation labeling tries to ascertain $\boldsymbol{P}_{\boldsymbol{i}}\left(\boldsymbol{l}_{\boldsymbol{k}}\right)$ through the following steps:

Initialization. Each region $\boldsymbol{r}_{\mathrm{i}}$ is initially assigned a set of probabilities, one for each query region $\boldsymbol{l}_{\boldsymbol{k}}$ that correspond to the similarity of their signatures (Sec. 2.2). Additionally, a compatibility matrix is established to take into account the relation between adjacent regions. Define the compatibility $\boldsymbol{C}_{i j}\left(\boldsymbol{l}_{\boldsymbol{k}}, \boldsymbol{l}_{\nu}\right)$ as the conditional probability that region $\boldsymbol{r}_{\boldsymbol{i}}$ correctly matches region $\boldsymbol{l}_{\boldsymbol{k}}$ in the query, given that a neighboring region $\boldsymbol{r}_{\boldsymbol{j}}$ is matched with region $\boldsymbol{l}_{\boldsymbol{l}}$ in the query.

Iteration. The probabilities $\boldsymbol{P}_{i}\left(\boldsymbol{l}_{k}\right)$ are iteratively updated according to the following change in likelihood:

$$
\delta P_{i}\left(l_{k}\right)=\sum_{j \in N} w_{i j}\left[\sum_{l \in L} C_{i j}\left(l_{k}, l_{l}\right) \cdot P_{j}\left(l_{l}\right)\right]
$$

where $N$ is the set of regions neighboring region $\boldsymbol{r}_{\boldsymbol{i}}$ and $W_{i j}$ are the weighting factors of these neighbors, such that $\Sigma w_{i j}=1$. The updated probabilities $\boldsymbol{P}_{\boldsymbol{i}}\left(\boldsymbol{l}_{\boldsymbol{k}}\right)^{(\boldsymbol{S}+1)}$ is computed by

$$
P_{i}\left(l_{k}\right)^{(S+1)}=\frac{P_{i}\left(l_{k}\right)^{(S)}\left[1+\delta P_{i}\left(l_{k}\right)^{(S)}\right]}{\sum_{l} P_{i}\left(l_{l}\right)^{(S)}\left[1+\delta P_{i}\left(l_{l}\right)^{(S)}\right]}
$$

The iterations continue until there is little or no change in the values of $\boldsymbol{P}_{i}\left(l_{k}\right)$.

Upon convergence, the values of $\boldsymbol{P}_{i}\left(\boldsymbol{l}_{k}\right)$ reflect probabilities of correct matches between regions of interest defined in the query, and the regions extracted from images in the library. Furthermore, since articulated objects in the scene can very well be segmented in a manner that corresponds to the object's individual parts, analyzing the segmented regions individually, then later composing the object according to its relational structure, 
facilitates articulated movement of object parts within the image.

\section{EXPERIMENTAL RESULTS}

Experiments were conducted on both static images and videos to test the validity of the proposed scheme. Images and videos from the library were prepared offline for efficiency. The first step was to extract the contours of individual regions by segmenting the scene using region growing. Anisotropic diffusion [4] was applied to facilitate smoothing and obtain better segmentation. Moreover, the contours were smoothed with a Gaussian filter to reduce noise. Finally, the individual affineinvariant signatures (Eq.1) and color information of each region was computed and stored in a database.

To test the scheme, queries were posed by identifying regions within a sample image. The database was then searched for matching regions using its signature and color information. The resulting probability matrix, which indicates how well each region matches with the query, was then used to initialize relaxation labeling in order to resolve any ambiguous matches.

In Figure 2, the top-left image is the sample query image. The object of interest was defined by the user and is shown in the next image as a set of extracted regions. The remaining images that are shown are frames that were retrieved from a three-minute video. The matching regions in the images are shown with heavy outlines. Notice the articulated movement of the different parts of the object, such as the arms. Figure 3 shows an example of the cross-correlation function defined by a pair of matching regions from the query and from the $5^{\text {th }}$ image. The peak in the graph indicates the phase shift where the signature match can be maximized. The figure on the right shows the corresponding signatures of the two regions. The consistency of the signatures indicates a strong similarity between the two regions.

\section{CONCLUSION}

We have presented a new method of retrieving images and video, based on the extraction and recognition of interesting regions of a segmented scene. The procedure is easy to implement, yet shows promising results in its ability to isolate interesting regions in images and video, allowing for a detailed examination of the scene in a way that is invariant to many visual transformations.

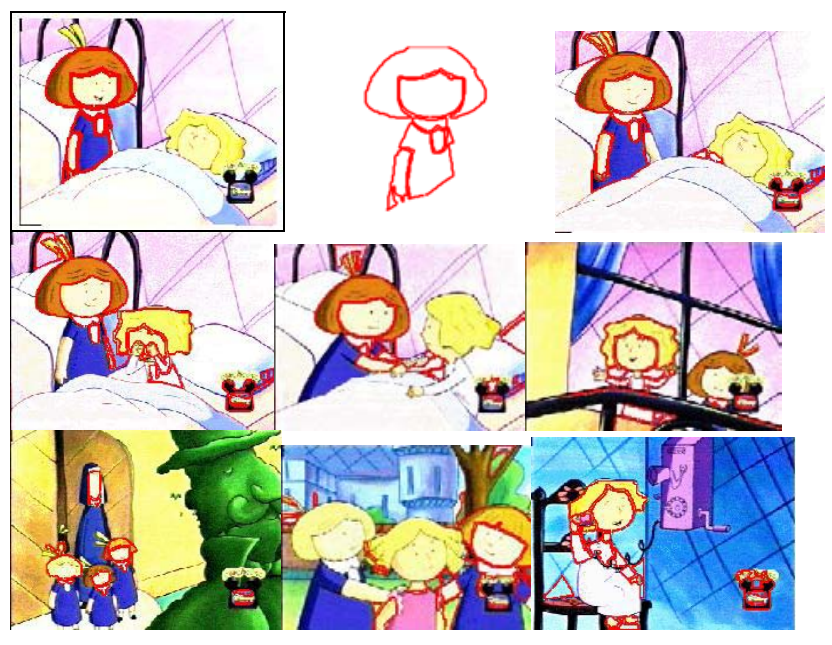

Figure 2. Frames retrieved from a video. The first two images are the queries, where the user identified regions of interest.
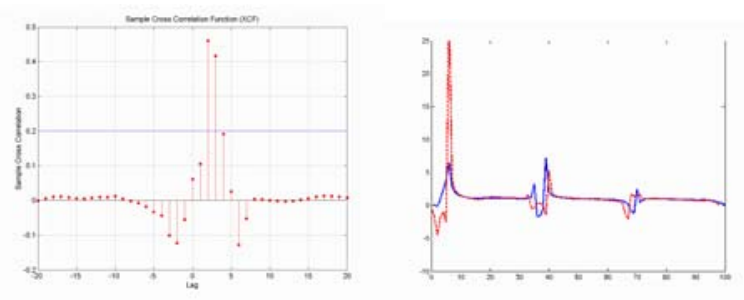

Figure 3. The cross correlation function (left) and the invariant signatures (right) of matching regions from Fig. 2.

Improvements to the technique include incorporating color information in matching regions whenever it is relevant, and a more sophisticated compatibility function can be used in the relaxation labeling stage, such as the relative orientation, size, and point of connection between regions.

\section{REFERENCES}

[1] R. Alferez and Y.F. Wang, "Geometric and Illumination Invariants for Object Recognition," IEEE Trans. on Pattern Analysis and Mach. Intelligence, vol. 21, pp. 505-536, 1999.

[2] K. Arbter, W.E. Snyder, H. Burkhardt and G. Hirzinger. "Application of Affine-Invariant Fourier Descriptors to Recognition of 3D Objects," IEEE Trans. PAMI, vol 12, pp. 640-647, 1990.

[3] H.W. Guggenheimer, Differential Geometry. New York: McGraw-Hill, 1963.

[4] P. Liang and Y.F. Wang, "Local Scale Controlled Anisotropic Diffusion with Local Noise Estimate for Image Smoothing and Edge Detection", submitted for publication in IEEE Transactions on Image Processing.

[5] T. Reiss. "Recognizing Planar Objects Using Invariant Image Features.” Springer-Verlag, Berlin, 1993. 